Research Paper:

\title{
Evaluating the Role of Pre-operative Cerebrospinal Fluid Diversion by Lumbar Drain in Transnasal Transsphenoidal Tumor Surgeries
}

\author{
Ajay Choudhary ${ }^{1}$, Rahul Varshney ${ }^{1^{*}}$, Pushkar Ranade $^{1}$, Kaviraj Kaushik ${ }^{1} \mathrm{Q}$
}

1. Department of Neurosurgery, PGIMER \& Dr. RML Hospital, New Delhi, India

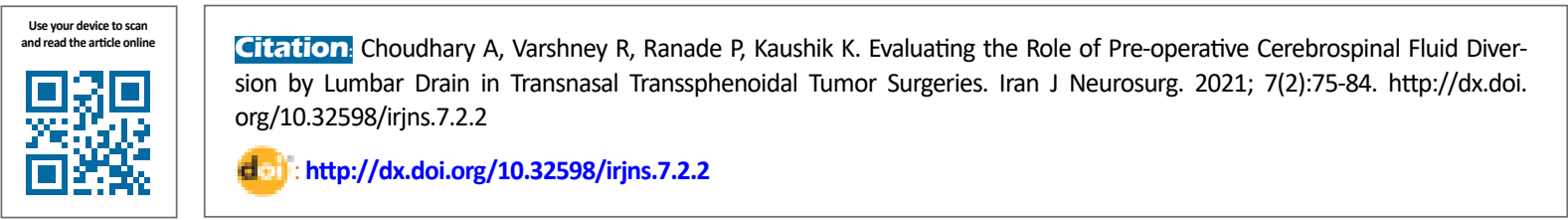

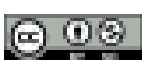

Article info:

Received: 10 Jan 2021

Accepted: 05 Mar 2021

Available Online: 01 Apr 2021

Keywords:

Endoscopic, Transsphenoidal, Cerebrospinal Fluid (CSF) leak, Meningitis, Rhinorrhea

\section{A B STRACT}

Background and Aim: The major concerns related to the Endoscopic Endonasal Transsphenoidal (EET) surgery for sellar and suprasellar tumors include the risks of post-operative Cerebrospinal Fluid (CSF) leak, leading to morbidity and at times mortality, due to severe meningitis. Time is required to develop possible preventive measures that can reduce the risk of post-operative CSF rhinorrhea. The present study aimed to evaluate the effects of pre-operative CSF diversion by lumbar drainage in EET tumor surgeries on preventing post-operative CSF leak and its effect on the length of hospital stay.

Methods and Materials/Patients: We conducted a prospective study on 20 patients with a pituitary tumor that underwent EET surgery between October 2018 and December 2019. Preoperative Lumbar Drain (LD) was inserted after induction in all explored patients. The tumor was excised with continuous intraoperative CSF drainage. Post-operatively, the LD was kept for 3 days and clamped for the next 24 hours. If no evidence of CSF rhinorrhea was present, it was removed. Complications related to CSF drainage, CSF leak, and hospital stays were evaluated.

Results: Our study population consisted of $13(65 \%)$ men and 7(35\%) women, with Mean $\pm S D$ age of $39.8 \pm 10.71$ years. The most commonly presented complaint was visual disturbance $(60 \%)$ and the least common symptom was urinary disturbance $(5 \%)$. The intra-operative leak was detected in $9(45 \%)$ patients, while the post-operative leak was present in only $1(5 \%)$ patient. LD blockage significantly contributed to post-operative CSF leak $(P=0.001)$. The Mean $\pm S D$ hospital stay in the post-operative period was $8.85 \pm 3.22$ days with $65 \%$ of patients having a hospital stay of $<7$ days. Other post-operative complications (e.g. diabetes insipidus, electrolyte imbalance, and hormonal disturbances) were mainly responsible for prolonged post-operative hospital stay $(P=0.001)$.

Conclusion: Pre-operative LD, apart from helping to reduce the incidence of post-operative CSF leak, is not associated with an overall increased post-operative hospital stay.

\section{*Corresponding Author:}

Rahul Varshney, MCh.

Address: Department of Neurosurgery, PGIMER \& Dr. RML Hospital, New Delhi, India

Tel: +98 (917) 890419212

E-mail: rahulvar1979@gmail.com 


\section{Highlights}

- Pre-operative lumbar drain in transnasal transsphenoidal surgeries for pituitary adenomas can aid surgical excision.

- It reduces the incidence of post-operative CSF leaks.

- It does not affect the length of hospital stay.

\section{Plain Language Summary}

There are various methods to operate tumors arising from a gland, called the pituitary gland inside the brain. One such promising technique is to remove it via an endoscope. It has the advantage of avoiding the opening of the skull and subsequent tumor removal. However, like other surgeries, it is associated with many complications. One of the most dreadful complications is the leakage of water-like fluid from the brain, called cerebrospinal fluid, which may lead to life-threatening complications, like meningitis. This study aimed to develop a technique to prevent or reduce the incidence of this post-operative CSF leak and its impact on hospital stay. We inserted a drain, called a lumbar drain, in the space present in the spinal cord to continuously drain the CSF from it. Post-operatively, this drain reduced the incidence of CSF leak; the results were comparable to other methods without complications associated with other techniques. Moreover, it did not increase hospital stay in our study. Thus, this method helps reduce the overall cost of treatment without major complications.

\section{Introduction}

$\mathrm{n}$ the modern era, Endoscopic Transnasal Transsphenoidal Surgery (ET-TS) is established as the standard practice for most sellar and suprasellar masses. It can be applied to any lesion within approximately $2 \mathrm{~cm}$ width of the midline skull base. Cerebrospinal fluid leakage is an unfortunate yet well-recognized complication of Transnasal Transsphenoidal Surgeries (TTS) [1-4]. It causes the bacterial contamination of Cerebrospinal Fluid (CSF) and predisposes patients to life-threatening meningitis. CSF leak can be observed intraoperatively, or in a delayed fashion, post-operatively. According to Jakimovski et al. [5], the rate of intraoperative CSF leak in endoscopic pituitary surgery with Intrathecal (IT) fluorescein administration was approximately $61 \%$. The intraoperative leak is a major risk factor for leaks in the post-operative period.

Persistent post-operative CSF leakage is the major cause of morbidity following transsphenoidal surgery [6-8]. Overall, the incidence of post-operative leaks is $0.5 \%-15 \%$ [9]. However, there are no clear-cut guidelines to manage CSF leaks following ET-TS [10]. CSF diversion through Lumbar Drain (LD) is an established practice to treat CSF leakage in cases that fail to respond to medical management. Lumbar drainage has been considered as a safe and effective treatment for CSF rhinorrhea following transsphenoidal surgery; however, this technique was not validated in prospective studies. According to
Alharbi et al. [11], pre-operative LD insertion is generally considered safe with a low risk of complications; however, it increases the length of hospitalization. Literature still lacks prospective studies concerning the advantages and disadvantages of pre-operative LD insertion in ET-TS surgeries. This study aimed to evaluate the advantages and disadvantages of LD in endoscopic TN-TS surgeries for sellar masses, especially for preventing intra-/postoperative CSF leak technique and its effect on the length of hospital stay.

\section{Methods and Materials/Patients}

From February 2018 to August 2019, 22 patients with pituitary adenoma were admitted to our hospital. Our study group consisted of only Indian subjects, aged 24-60 years presenting with sellar tumor and positive Magnetic Resonance Imaging (MRI) data confirming the diagnosis. Out of the 22 patients with sellar tumor, 2 subjects refused surgery; accordingly, they were excluded from our study. Thus, 20 patients were finally included (13 males and 7 females). Sellar tumor patients were diagnosed based on clinical examination and neuroimaging findings according to pituitary protocol with Non-Contrast CT Scan (NCCT) of head and CT Scan of Paranasal Sinuses (CT-PNS); based on which, patients with active sinusitis, kissing carotids, and Middle Cranial Fossa (MCF) extensions were excluded from the study. The Institutional Ethics Committee (Ethics Committee, PGIMER, Dr. RML Hospital, New Delhi, Registration no 
ECR/78/Inst/DL/2013/RR-19 issued under New Drugs and Clinical Trials Rules, 2019) approved this study. Furthermore, the research was performed as per the relevant guidelines issued by PGIMER and RML Hospital, New Delhi. Written informed consent was obtained from all study participants or their legal guardians.

Pre-operative LD placement was conducted in all patients inside the operation theatre after induction. Following intubation, in lateral decubitus position, the catheter was inserted into the L4-5 inter-laminar space using a 14-gauge Tuohy needle, and connected to a closed drainage system.

Tumor resection: trans-sphenoidal surgery was performed for the excision of sellar tumors with parasellar and suprasellar extensions through the right nostril. Middle turbinate was laterally pushed to obtain further surgical freedom. Next, the endoscope was angled upwards, about $1.5 \mathrm{~cm}$, to reach the sphenoid ostium. The sellar floor was drilled out and the dura was opened in a cruciate manner. The tumor present just over the dura was resected in a standard manner. After satisfactory tumor removal, dural defect sealing was conducted using the naso-septal flap, with or without glue and autologous graft with fat in cases with intra-op CSF leaks. Nasal packing was post-operatively performed and removed on the third day.

CSF drainage: continuous CSF drainage was intraoperatively conducted. Post-operative LD remained at the shoulder level and was allowed to drain 10-15 cc/ hour under close monitoring, for a maximum of 120 $150 \mathrm{cc} /$ day for the first 3 days followed by clamping for the next 24 hours. If no evidence of CSF rhinorrhea was found, LD was removed. The complications of the CSF drainage, LD use, and its effects on tumor resection, as well as CSF leak and hospital stay, were evaluated. Data analysis was conducted using SPSS version 27 . The significance level of all of the statistical analyses was $95 \%$.

\section{Results}

The study participants consisted of 20 individuals; $7(35 \%)$ females and $13(65 \%)$ males, with a Mean \pm SD age of $39.80 \pm 10.71$ years. All study patients presented pituitary adenoma on histopathology. The majority of the tumors were non-functional (70\%). The most common functional types were $\mathrm{GH}$-secreting adenomas (66.66\%), followed by prolactin-secreting adenomas (33.33\%). The most common presenting symptoms included visual disturbance (60\%) followed by headache (55\%) (Figure 1 ).

On pre-operative MRI imaging, the suprasellar extension was detected in $70 \%$ of the cases; parasellar extension and internal carotid artery involvement were each observed in $90 \%$ of the explored cases (Table 1 ). Out of a total of 20 examined patients, the intra-operative leak was observed in $9(45 \%)$ patients, while the post-operative leak was present in only $1(5 \%)$ patient (Table 2 ).

Tumor extensions and the functioning status of the tumor did not significantly affect the incidence of the intra-operative leak (Table 3). The most common complaint after lumbar drainage was headache (65\% of patients) mostly on the second post-op day, followed by local site pain in $60 \%$ of the study patients. Furthermore, $15 \%$ of the study subjects reported bilateral lower limb pain. Drain blockage was observed in $2(10 \%)$ patients (Figure 2). Out of which, one presented intra-operative and post-operative drain blockage, and 1 subject had

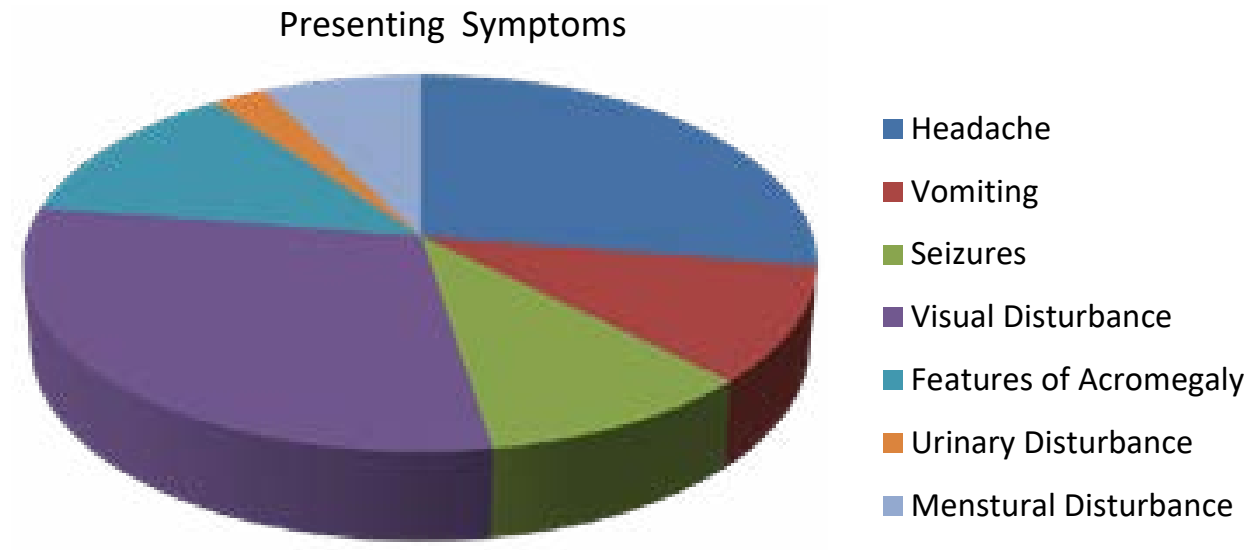

Figure 1. The presenting symptoms in the study group 
Table 1. A summary of clinical characteristics and CSF leak in 20 cases treated by the resection of pituitary adenoma

\begin{tabular}{|c|c|c|c|c|}
\hline \multicolumn{2}{|c|}{ Features } & No. & Intra-op & Post-op \\
\hline \multirow{3}{*}{ Gender } & Male & 13 & 7 & 1 \\
\hline & & & & \\
\hline & Female & 7 & 2 & 0 \\
\hline \multirow{3}{*}{ Age } & $<50$ years & 14 & 6 & 0 \\
\hline & & & & \\
\hline & $\geq 50$ years & 6 & 3 & 1 \\
\hline \multirow{5}{*}{ Tumor type } & Non-functioning & 14 & 5 & 0 \\
\hline & $\mathrm{GH}$ & 4 & 3 & 1 \\
\hline & & & & \\
\hline & PRL & 2 & 1 & 0 \\
\hline & Others & 0 & 0 & 0 \\
\hline \multirow{3}{*}{ Tumor size } & Microadenoma & 0 & 0 & 0 \\
\hline & & & & \\
\hline & Macroadenoma & 20 & 9 & 1 \\
\hline \multirow{3}{*}{ Parasellar extension } & Right & 11 & 4 & 0 \\
\hline & Left & 6 & 7 & 0 \\
\hline & Both & 3 & 2 & 1 \\
\hline \multirow{2}{*}{ Surprasellar extension } & Present & 14 & 8 & 1 \\
\hline & Absent & 6 & 1 & 0 \\
\hline \multirow{5}{*}{ Knosp grade } & 0 & 2 & 0 & 0 \\
\hline & 1 & 7 & 3 & 0 \\
\hline & 2 & 7 & 3 & 0 \\
\hline & 3 & 3 & 2 & 0 \\
\hline & 4 & 1 & 1 & 1 \\
\hline
\end{tabular}

Optic chiasma

\begin{tabular}{ccccc} 
Displacement & Present & 14 & 8 & 1 \\
& Absent & 6 & 1 & 0 \\
The extent of resection & Gross total resection & 16 & 7 & 1 \\
\hline & Subtotal resection & 4 & 2 & 1 \\
\hline
\end{tabular}

only post-operative drain blockage. One patient also developed meningitis, who was successfully treated with intravenous antibiotics.

On statistical analysis, LD blockage was found as a significant cause of post-operative CSF leak ( $P=0.001)$. The intra-operative leak was a significant factor causing post-operative headaches $(P=0.045)$ (Table 4).
The Mean $\pm S D$ post-operative hospital stay was $8.85 \pm 3.22$ days with $65 \%$ of the explored patients having hospital stay of $<7$ days. Minor LD complications such as headache and local site pain were common; however, they were not associated with a prolonged hospital stay. Meningitis, in one patient, significantly prolonged postop hospital stay (Table 5). Other post-operative complications (e.g. diabetes insipidus, electrolyte imbalance, and 


\section{Lumbar Drain Complications}

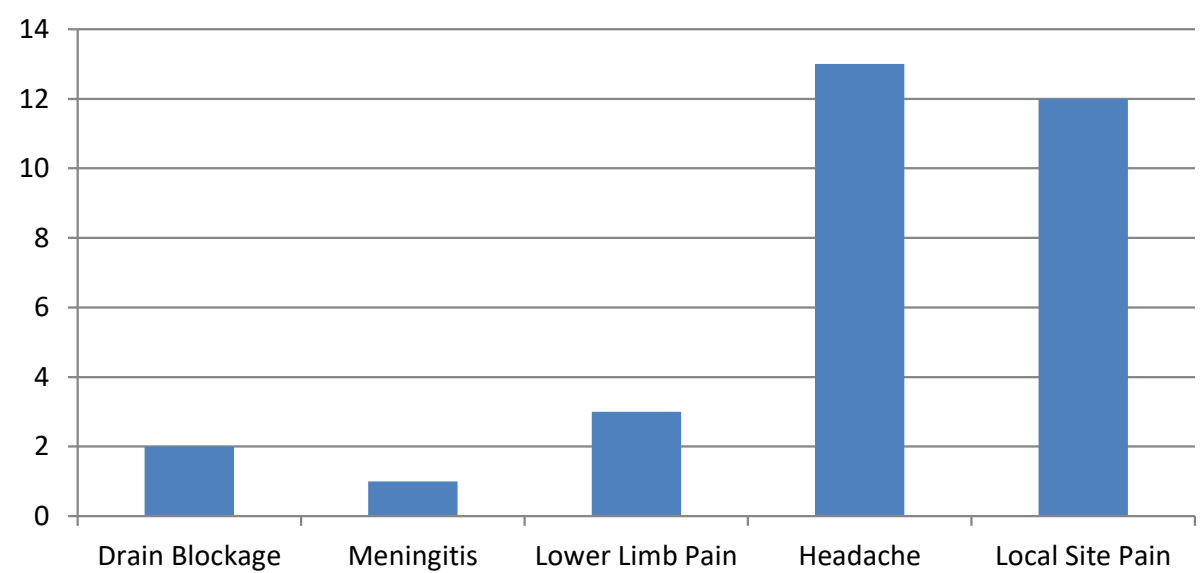

Figure 2. Post-operative lumbar drain complications

hormonal disturbances) were mainly responsible for prolonged post-operative hospital stay $(\mathrm{P}=0.001)$ (Table 6).

Vision improvement and the resolution of headache were detected in $50 \%$ of the examined patients; hormonal imbalance improved in $15 \%$ of cases. Seizures were resolved in $15 \%$ of the investigated patients, while 2 patients with the features of acromegaly encountered persistent symptoms. There was a significant improvement in symptoms, such as headache, seizures, and vision impairments after surgery $(P<0.05)$.

\section{Discussion}

Transsphenoidal surgery for sellar tumors is considered the standard approach, especially for pituitary macroadenomas. CSF rhinorrhea following surgery remains a major complication leading to increased morbidity. This condition requires further measures to reduce CSF leak by medical management or surgical procedures; subsequently, this process increases the cost of management, hospital stay, and affects patients' therapeutic outcomes. Various studies indicated that the incidence of post-oper- ative CSF leak is higher in patients with intraoperative CSF $[12,13]$; no definitive techniques have been described in the literature to reduce the incidence of CSF leak. The sellar diaphragm is typically expanded by pituitary macroadenomas, exposing the arachnoid to intraoperative injury and CSF leakage; thus, the intraoperative drainage of CSF reduces tension on the arachnoid, making it less susceptible to puncture [14]. Pre-operative drainage is presumed to help decrease intracranial pressure and brain swelling by external CSF diversion. This condition results in enhanced access to the tumor or the floor of the anterior cranial fossa. This facilitates tumor resection with minimal injury to arachnoid and diaphragm sellae and minimizes the chances of CSF leak. Consequently, it promotes dural repair healing, post-operatively. LD also facilitates the fall of the tumor and reduces tumor turgor and elevates the volume of tumor resection. CSF drainage might be of particular utility in the resection of macroadenomas with suprasellar extension. The majority of the tumor may be bounded only by a very thin layer of the normal gland or by arachnoid. LD is also associated with few complications, such as over drainage syndromes and infections, i.e., noted with continuous drainage method

Table 2. Incidence of CSF leak intraoperatively and postoperatively

\begin{tabular}{lcc}
\hline CSF Leak & No. (\%) & Post-op \\
\cline { 2 - 3 } & Intra-op & $1(5.0)$ \\
Present & $9(45.0)$ & $19(95.0)$ \\
\hline Absent & $11(55.0)$ & \\
\hline
\end{tabular}


Table 3. The correlation of intra-op leak with risk factors

\begin{tabular}{|c|c|c|}
\hline Risk Factors & Correlation Coefficient & $\mathbf{P}$ \\
\hline \multirow{3}{*}{ Suprasellar extension } & $\mathrm{R}$ value & 0.154 \\
\hline & & \\
\hline & $P$ & 0.518 \\
\hline \multirow{3}{*}{ Internal carotid artery } & $\mathrm{R}$ value & 0.264 \\
\hline & & \\
\hline & $P$ & 0.290 \\
\hline \multirow{3}{*}{ Functioning tumors } & $\mathrm{R}$ value & 0.285 \\
\hline & & \\
\hline & $\mathrm{P}$ & 0.223 \\
\hline \multirow{3}{*}{ Non-functioning tumor } & $\mathrm{R}$ value & 0.285 \\
\hline & & \\
\hline & $P$ & 0.223 \\
\hline \multirow{3}{*}{ GH-secreting tumor } & $\mathrm{R}$ value & 0.406 \\
\hline & & \\
\hline & $P$ & 0.076 \\
\hline \multirow{3}{*}{ Post-operative residual } & $\mathrm{R}$ value & 0.066 \\
\hline & & \\
\hline & $P$ & 0.783 \\
\hline
\end{tabular}

$[4,15]$. Therefore, LD placement prior to elective surgery remains controversial.

We also observed that surgery for recurrent/residual tumors had increased the incidence of intra-operative CSF leak; functional status also has more propensity for the intra-operative leak, compared to non-functional status. However, none of the factors including tumor histopathology and tumor extensions significantly affect the intra-operative leak. Moreover, 9 out of the 20(45\%) explored patients presented an intraoperative CSF leak, i.e., comparable to a large body of literature where the

Table 4. Correlations between LD complications and CSF leak

\begin{tabular}{|c|c|c|c|}
\hline Risk Factors & & CSFLEAKINTRAOP & CSFLEAKPOSTOP \\
\hline \multirow[b]{2}{*}{ Blockage } & $R$ value & 0.034 & 0.688 \\
\hline & $P$ value & 0.888 & 0.001 \\
\hline \multirow{2}{*}{ Local infection } & $R$ value & 0 & 0 \\
\hline & $P$ value & 0 & 0 \\
\hline \multirow{2}{*}{ Meningitis } & $R$ value & 0.254 & -0.053 \\
\hline & $P$ value & 0.281 & 0.826 \\
\hline \multirow{2}{*}{ Lower limb pain } & $R$ value & -0.380 & -0.096 \\
\hline & $P$ value & 0.098 & 0.686 \\
\hline \multirow{2}{*}{ Headache complication } & $R$ value & 0.453 & 0.168 \\
\hline & $P$ value & 0.045 & 0.478 \\
\hline
\end{tabular}

$R$ value: correlation coefficient. 
Table 5. Correlations between lumbar drain complications and post-operative hospital stay

\begin{tabular}{ccc}
\hline Lumbar Drain Complications & Correlation Coefficient & P \\
\hline Blockage & 0.303 & 0.194 \\
Meningitis & 0.450 & 0.046 \\
Lower limb pain & 0.337 & 0.024 \\
Headache & -0.503 & 0.059 \\
Local site pain or backache & 0.430 & \\
\hline
\end{tabular}

lumbar drain was not pre-operatively used; in such cases, the associated risk approximately equaled $50 \%$. El Shazly et al. [16] reported a $73.9 \%$ incidence of intra-operative leaks among 23 examined patients; however, in some studies, the incidence was observed as low as $17.9 \%$ [17].

In our study, pre-operative LD insertion was not correlated with a significant decrease in the incidence of intraoperative CSF leak. Other characteristics, such as suprasellar extension [18], recurrent surgery [19], the functioning status of the tumor, and aggressive surgical resection [19], may impact intraoperative leak. Thus, the assessment of these factors requires a large-scale study with a control group design.

Time was required to develop ideal preventive measures that minimize the odds of intra-operative as well as postoperative CSF leaks and various complications associated with those. Hong et al. [20] reported that the incidence of post-operative CSF leak was calculated as $9.3 \%$ when applying the conventional method of sella floor repair.

According to Wang et al. [21] using gelatin sponge and hydrogel sealant overlay to prevent low-grade post-operative CSF leaks were safe and comparable in efficacy, whilst avoiding the morbidity related to more complex sellar reconstructions. However, this gelatin sponge serves as a potential nidus of infection and subsequent abscess formation. Moreover, it is only beneficial in lowgrade (grade 1) CSF leaks; its efficacy in high-grade leaks remains unclear.
Attempts were made for sellar floor reconstruction with numerous synthetic materials, such asbone [22], ceramic [23], and titanium [24], as an adjunct to autologous fat muscle or fascia; however, these foreign bodies may also cause a host reaction and serve as a nidus for infection or complicate post-operative imaging. Artifacts observed on MRI were reported with stainless steel [24] and titanium [25].

Sharifi et al. [26] advocated the bipolar coagulation of prolapsed arachnoid in 13 patients to prevent post-operative CSF leaks. During the post-operative follow-up period, none of the explored patients experienced early or delayed post-operative CSF leakage. However, sometimes, the defect is very slight and at times, not visible; thus, the bipolar coagulation of the arachnoid may not lead to the closure of the defect. Moreover, bipolar coagulation carries the risk although less, but definite, injury to the surrounding neural tissue. The sample size was small and requires a large randomized controlled trial to validate the effectiveness of arachnoid coagulation and its potential associated complications.

The conventional method of intraoperative CSF leak repair by fibrin sealants with or without fascia and autologous fat graft also presents certain disadvantages. It includes time-consuming preparation, also these sealants can precipitate acute immune responses; chronically, they are associated with adhesion formation and infection [27].

Table 6. Post-operative stay and post-operative complications

\begin{tabular}{|c|c|c|c|}
\hline Post-op Complications & Mean \pm SD & $\mathbf{t}$ & $\mathbf{P}$ \\
\hline Present & $10.73 \pm 3.29$ & & \\
\hline & & 3.74 & 0.001 \\
\hline Absent & $6.56 \pm 0.53$ & & \\
\hline
\end{tabular}


In a meta-analysis by Tan et al. [28] covering 678 cases, the incidence of intraoperative CSF leakage was $12.4 \%$ in the intraoperative LD group and $39.3 \%$ in the intraoperative non-LD group. Post-operative CSF leakage was measured to be $2.9 \%$ in the intraoperative LD group, and $6.3 \%$ in the intraoperative non-LD group. Thus, a significant difference was present in favor of intraoperative LD.

Sade et al. [29] also recommended pre-operative LD insertion before the procedure, especially in adenomas with suprasellar extension. As an adjunctive benefit, this allows for the saline-infusion method to mobilize the suprasellar part without producing the venous engorgement of the Valsalva maneuver. Contrarily, Caggiano et al. [30] concluded that intraoperative LD was impractical in preventing intraoperative and post-operative CSF leakage.

In our study, the incidence of intraoperative CSF leak was not significantly reduced; however, pre-operative CSF drainage, using LD significantly reduced post-operative leak (1 leak out of 20). The patient who manifested post-operative CSF leak drain blockage was a significant contributory factor for CSF rhinorrhea $(P<0.001)$. Our results were consistent with those of Sada et al. [29] and Ransom et al. [14] where the incidence of the post-operative leak was reported as $1.6 \%$ and $6 \%$, respectively.

We observed that symptoms such as headache, seizure episodes, and visual disturbances had significantly improved, indicating adequate tumor excision. However, post-operative imaging illustrated tumor residual in 4 patients. Pre-operative CSF drainage helps with adequate tumor excision with the resolution of presenting symptoms; however, tumor extension to cavernous sinus or the encasement of the internal carotid artery might hamper complete tumor removal despite adequate tumor fall with pre-operative drainage.

Lumbar CSF drainage has been found to be useful but there is a paucity of level 1 evidence, indicating its usage. Although the complication rate is lower, the severity of complications differs in lumbar drainage. Understanding these complications is necessary to decrease morbidity and save healthcare costs. Most examined patients mainly developed minor complications due to lumbar drainage such as headache and backache, which were managed by oral or parenteral medications. Kitchel et al. [2] reported a $58 \%$ incidence rate for transient headache during active drainage, i.e., similar to the $63 \%$ incidence rate reported by Shapiro and associates [4].

Symptoms may resolve in 48 hours or may persist for one or more weeks. Overall, the obtained data suggested that patients with minor lumbar drain complications (headache being most common), experienced no prolonged hospital stay, compared to the patients without complications $(P<0.05)$. Accidental over-drainage is a significant contributor to complications with LDs. Pneumocephalus, subdural hemorrhage, neurological decline, or uncal herniation are potential relevant complications. However, none of these conditions were detected in our study following lumbar drainage. The rate of drainage should not exceed $5-10 \mathrm{~mL} / \mathrm{h}$ (normal adult CSF production is approximately $20 \mathrm{~mL} / \mathrm{h}$ ); therefore, eliminating a pressure gradient that could lead to pneumocephalus [31].

Alharbi et al. [11] concluded that LD use had led to an increased hospital stay, compared to patients without lumbar drainage $(P<0.001)$. The most common causes for prolonged hospitalization in the LD group consisted of headache, nasal bleeding, and developing post-operative CSF leak. In those who did not receive LD, the most frequent causes of prolonged hospital stay were diabetes insipidus, electrolyte abnormalities, CSF leak, and Deep Vein Thrombosis (DVT). Jung et al. [10] concluded that LD use increased the length of hospital stay. The present study findings however differed from those of the abovementioned studies, suggesting a lower mean hospital stay in patients with pre-operative lumbar drainage; it significantly increases if major complications of lumbar drainage, like meningitis, occur or postoperative complications develop.

Pre-operative lumbar drain helps to avoid further surgery for CSF leak resulting in decreased healthcare costs. Coupled with improved and accurate intraoperative CSF leak detection methods, it can reduce skull base reconstruction pressure, especially for young physicians. The results were comparable with other methods to reduce the incidence of post-operative CSF leaks. As an adjunctive benefit, this allows for the saline-infusion method to mobilize the extended suprasellar part, preventing venous engorgement due to the Valsalva maneuver. Accordingly, we suggest that LD be pre-operatively used during transsphenoidal tumor surgery to reduce postoperative CSF leak for better therapeutic outcomes.

The present study had some limitations. The study sample size was limited to have conclusive evidence about the possible role of pre-operative LD in preventing intraoperative and post-operative CSF leaks. This warrants the need for a large-scale prospective randomized case-control study. The exact incidence of intraoperative CSF leak cannot be estimated, because reduced tension on the arachnoid may conceal the small arachnoid tear; thus affecting the judgment of CSF leak. Elevated body 
mass index is a risk factor for post-operative CSF leakage and we lacked data regarding obesity in our study.

\section{Conclusion}

The data of the present study indicated that LD not only helps to reduce the incidence of post-operative CSF leak following transsphenoidal surgery but also is beneficial with adequate tumor excision, resulting in the significant resolution of the patient's symptoms and better therapeutic outcomes. Pre-operative lumbar drainage was not associated with an overall increased post-operative hospital stay.

\section{Ethical Considerations}

\section{Compliance with ethical guidelines}

Institutional ethical committee (Ethics Committee, PGIMER, Dr. RML Hospital, New Delhi, Registration no ECR/78/Inst/DL/2013/RR-19 issued under New Drugs and Clinical Trials Rules, 2019) approval was taken. All the research steps were performed following relevant guidelines issued by PGIMER \& Dr.RML Hospital, New Delhi. Written informed consent was obtained from all participants and their legal guardians.

\section{Funding}

This research did not receive any grant from funding agencies in the public, commercial, or non-profit sectors.

\section{Authors' contributions}

Conception and design: Ajay Choudhary; Drafting the article: Rahul Varshney; Data collection: Pushkar Ranade; Data analysis and interpretation: Kaviraj Kaushik; Critically revising: Rahul Varshney; Reviewing the submitted version of the manuscript: Pushkar Ranade.

\section{Conflict of interest}

The authors declare that there is no conflict of interest.

\section{References}

[1] Coiteiro D, Távora L, Antunes JL. Spontaneous cerebrospinal fluid fistula through the clivus: Report of two cases. Neurosurgery. 1995; 37(4):826-8. [DOI:10.1097/00006123199510000-00030] [PMID]
[2] Kitchel SH, Eismont FJ, Green BA. Closed subarachnoid drainage for management of cerebrospinal fluid leakage after an operation on the spine. The Journal of Bone \& Joint Surgery. American Volume. 1989; 71(7):984-7. [DOI:10.2106/00004623198971070-00004] [PMID]

[3] McCallum J, Maroon JC, Jannetta PJ. Treatment of postoperative cerebrospinal fluid fistulas by subarachnoid drainage. Journal of Neurosurgery. 1975; 42(4):434-7. [DOI:10.3171/ jns.1975.42.4.0434] [PMID]

[4] Shapiro SA, Scully T. Closed continuous drainage of cerebrospinal fluid via a lumbar subarachnoid catheter for treatment or prevention of cranial/spinal cerebrospinal fluid fistula. Neurosurgery 1992; 30(2):241-5. [DOI:10.1227/00006123-199202000-00015] [PMID]

[5] Jakimovski D, Bonci G, Attia M, Shao H, Hofstetter C, Tsiouris AJ, et al. Incidence and significance of intraoperative cerebrospinal fluid leak in endoscopic pituitary surgery using intrathecal fluorescein. World Neurosurgery. 2014; 82(34):e513-23. [DOI:10.1016/j.wneu.2013.06.005] [PMID]

[6] Benveniste RJ, King WA, Walsh J, Lee JS, Delman BA, Post KD. Repeated transsphenoidal surgery to treat recurrent or residual pituitary adenoma. Journal of Neurosurgery. 2005; 102(6):1004-12. [DOI:10.3171/jns.2005.102.6.1004] [PMID]

[7] Black PM, Zervas NT, Candia GL. Incidence and management of complications of transsphenoidal operation for pituitary adenomas. Neurosurgery. 1987; 20(6):920-4. [DOI:10.1227/00006123-198706000-00017] [PMID]

[8] Zada G, Kelly DF, Cohan P, Wang C, Swerdloff R. Endonasal transsphenoidal approach for pituitary adenomas and other sellar lesions: An assessment of efficacy, safety, and patient impressions. Journal of Neurosurgery. 2003; 98(2):350-8. [DOI:10.3171/jns.2003.98.2.0350] [PMID]

[9] Shiley SG, Limonadi F, Delashaw JB, Barnwell SL, Andersen PE, Hwang PH, et al. Incidence, etiology, and management of cerebrospinal fluid leaks following trans-sphenoidal surgery. The Laryngoscope. 2003; 113(8):1283-8. [DOI:10.1097/00005537-200308000-00003] [PMID]

[10] Jung H, Shah A, Ajlan A. Perioperative cerebrospinal fluid diversion utilizing lumbar drains in transsphenoidal surgery. Neurological Disorders. 2014; 2(2):1000150. [DOI:10.4172/2329-6895.1000150]

[11] AlharbiS, Harsh G, Ajlan A. Perioperative lumbar drain utilization in transsphenoidal pituitary resection. Neurosciences. 2018; 23(1):46-51. [DOI:10.17712/nsj.2018.1.20170136] [PMID] [PMCID]

[12] Phogat V, Meena US, Mishra K, Sharma A. Post-operative cerebrospinal fluid leak following transsphenoidal pituitary surgery: Predictive factors and management options- A Single-institute experience. International Journal of Scientific Study. 2021; 8(10):66-70._http://www.ijss-sn.com/uploads/2/0/1/5/20153321/13_ijss_jan_oa_08_-_2021.pdf

[13] Shiley SG, Limonadi F, Delashaw JB, Barnwell SL, Andersen PE, Hwang PH, et al. Incidence, etiology, and management of cerebrospinal fluid leaks following trans-sphenoidal surgery. Laryngoscope. 2003; 113(8):1283-8. [DOI: 10.1097/00005537-200308000-00003] [PMID]

[14] Ransom ER, Palmer JN, Kennedy DW, Chiu AG. Assessing risk/benefit of lumbar drain use for endoscopic skull-base 
surgery. International Forum of Allergy \& Rhinology. 2011; 1(3):173-7. [DOI:10.1002/alr.20026] [PMID]

[15] Cappabianca P, Cavallo LM, Valente V, Romano I, D'Enza AI, Esposito F, et al. Sellar repair with fibrin sealant and collagen fleece after endoscopic endonasal transsphenoidal surgery. Surgical Neurology. 2004; 62(3):227-33. [DOI:10.1016/j. surneu.2004.01.016] [PMID]

[16] El Shazly AA, El Wardany MA, Abo El Ezz TA. Sellar repair with autologous muscle and composite septal cartilage grafts for treatment of cerebrospinal fluid leakage following trans-sphenoidal pituitary surgery. Asian Journal of Neurosurgery. 2016; 11(4):433-9. [DOI:10.4103/1793-5482.144157] [PMID] [PMCID]

[17] Zhang C, Ding X, Lu Y, Hu L, Hu G. Cerebrospinal fluid rhinorrhoea following transsphenoidal surgery for pituitary adenoma: Experience in a Chinese centre. Acta Otorhinolaryngologica Italica. 2017; 37(4):303-7. [DOI:10.14639/0392100X-1086] [PMID] [PMCID]

[18] Patel PN, Stafford AM, Patrinely JR, Smith DK, Turner JH, Russell PT, et al. Risk factors for intraoperative and postoperative cerebrospinal fluid leaks in endoscopic transsphenoidal sellar surgery. Otolaryngology-Head and Neck Surgery. 2018; 158(5):952-60. [DOI:10.1177/0194599818756272] [PMID]

[19] Han ZL, He DS, Mao ZG, Wang HJ. Cerebrospinal fluid rhinorrhea following trans-sphenoidal pituitary macroadenoma surgery: Experience from 592 patients. Clinical Neurology and Neurosurgery. 2008; 110(6):570-9. [DOI:10.1016/j. clineuro.2008.02.017] [PMID]

[20] Hong CK, Kim YB, Hong JB, Lee KS. Sealing of cerebrospinal fluid leakage during conventional transsphenoidal surgery using a fibrin-coated collagen fleece. Journal of Clinical Neuroscience. 2015; 22(4):696-9. [DOI:10.1016/j. jocn.2014.10.019] [PMID]

[21] Wang YY, Kearney T, Gnanalingham KK. Low-grade CSF leaks in endoscopic trans-sphenoidal pituitary surgery: Efficacy of a simple and fully synthetic repair with a hydrogel sealant. Acta Neurochirurgica. 2011; 153(4):815-22. [DOI:10.1007/ s00701-010-0862-8] [PMID]

[22] Hardy J, Cutcheon MC. IE: Pituitary microadenomas. In Apuzzo MLJ, editor. Brain surgery: Complication avoidance and management. New York: Churchill Livingstone; 1993.

[23] Kobayashi S, Sugita K, Matsuo K, Inoue T. Reconstruction of the sellar floor during transsphenoidal operations using alumina ceramic. Surgical Neurology. 1981; 15(3):196-7. [DOI:10.1016/0090-3019(81)90142-7] [PMID]

[24] Arita K, Kurisu K, Tominaga A, Ikawa F, Iida K, Hama S, et al. Size-adjustable titanium plate for reconstruction of the sella turcica. Technical note. Journal of Neurosurgery. 1999; 91(6):1055-7. [DOI:10.3171/jns.1999.91.6.1055] [PMID]

[25] FreidbergSR, Hybels RL, Bohigian RK. Closure of cerebrospinal fluid leakage after transsphenoidal surgery: Technical note. Neurosurgery. 1994; 35(1):159-60. [DOI:10.1227/00006123199407000-00027] [PMID]

[26] Sharifi G, Akbari Dilmaghani N, Sadrhosseini SM, Arastou $\mathrm{S}$. Arachnoid prolapse in endoscopic transsphenoidal surgery of pituitary adenoma, technical note. British Journal of Neurosurgery. 2020; 1-7. [DOI:10.1080/02688697.2020.1779178] [PMID]
[27] Grotenhuis JA. Costs of postoperative cerebrospinal fluid leakage: 1- year, retrospective analysis of 412 consecutive nontrauma cases. Surgical Neurology. 2005; 64(6):490-3. [DOI:10.1016/j.surneu.2005.03.041] [PMID]

[28] Tan J, Song R, Huan R, Huang N, Chen J. Intraoperative lumbar drainage can prevent cerebrospinal fluid leakage during transsphenoidal surgery for pituitary adenomas: A systematic review and meta-analysis. BMC Neurology. 2020; 20(1):303. [DOI:10.1186/s12883-020-01877-z] [PMID] [PMCID]

[29] Sade B, Mohr G, Frenkiel S. Management of intra-operative cerebrospinal fluid leak in transnasal transsphenoidal pituitary microsurgery: Use of post-operative lumbar drain and sellar reconstruction without fat packing. Acta Neurochirurgica. 2006; 148(1):13-9. [DOI:10.1007/s00701-005-0664-6] [PMID]

[30] Caggiano C, Penn DL, Laws Jr ER. The role of the lumbar drain in endoscopic endonasal skull base surgery: A retrospective analysis of 811 cases. World Neurosurgery. 2018; 117:e575-9. [DOI:10.1016/j.wneu.2018.06.090] [PMID]

[31] Açikbaş SC, Akyüz M, Kazan S, Tuncer R. Complications of closed continuous lumbar drainage of cerebrospinal fluid. Acta Neurochirurgica. 2002; 144(5):475-80. [DOI:10.1007/ s007010200068] [PMID] 